Case Report

\title{
A Case Report of a Robotic Thoracosopic Parathyroidectomy in a Community Hospital
}

\author{
Emily Ann Harris, $M D^{1^{*}}$, Shabirhusain S Abadin, MD, MPH, FACS ${ }^{2}$ and Keith D Bowersox, MD, PhD \\ ${ }^{1}$ Department of General Surgery, AMITA Saint Joseph Hospital, USA \\ ${ }^{2}$ Endocrine Surgeon, AMITA Saint Joseph Hospital, USA \\ ${ }^{3}$ Cardiothoracic Surgeon, Rush University Medical Center, USA
}

\begin{abstract}
Mediastinal parathyroid adenomas pose a technical challenge to surgeons, as they exist in remote and difficult to access locations. Historically, approaches using sternotomy, thoracotomy, and even VATS, have been implemented. However, recently the da Vinci Robotic surgical systems have gained popularity for resection of these lesions. In this case report, we document an incident where the da Vinci Robot is used to resect an aortopulmonary window parathyroid adenoma. This case report sheds light on both the feasibility and practicality of using the da Vinci Robot for resection of parathyroid adenomas, as well as the translatability of implementing robotic approaches in the community hospital setting.
\end{abstract}

Keywords

Parathyroid, Robotic, Aortopulmonary window

\section{Introduction}

Historically, $15-20 \%$ of patients with hyperparathyroidism have ectopic parathyroid glands [1-3], which are due to abnormal migration during embryogenesis and can be located anywhere from the base of the tongue to the mediastinum [4]. Notably, less than 1-3\% existing of these ectopic parathyroid glands exist deep within the mediastinum [1,3], and can be an important cause of persistent or primary hyperparathyroidism [4].

Unfortunately, these deep mediastinal lesions present a surgical challenge, as they exist in difficult to access locations which are not surgically accessible with a traditional low cervical incision $[2,4,5]$, thereby requiring sternotomy or thoracotomy [1]; both of which are associated with significant morbidity $[2,5]$. Although video-assisted thoracoscopic surgery (VATS) has been utilized widely for lung and esophageal pathologies, much less it written on its utility in mediastinal parathyroid surgery. Therefore, recently minimally invasive techniques using the da Vinci Robotic surgical systems have been under evaluation for resection ectopic mediastinal parathyroid adenomas [3]. The robotic platform provides more dexterity and better visualization than the VATS approach [3].

The benefits of the robot approach over the conventional open and VATs approaches include the following: Less morbidity, three-dimensional optics, better precision and mobility with endowrist instruments, a stable camera platform, and better ergonomics for the surgeon $[1,3,6,7]$. All of these improvements and benefits allow for better identification and dissection of ectopic parathyroid tissue as well as accessing small and remote surgical fields $[1,6]$, which are necessary features when resecting mediastinal parathyroid adenomas.

There are several case reports of the robotic assisted approach for the resection of mediastinal parathyroid adenomas $[1,6,7]$, with the report of the first of this surgery in 2004 by Bodner [8].

Specifically, as in this case report, the resection of a parathyroid adenoma from the aortopulmonary window represents an ideal case for robotic surgery with two prior case reports of this documented in the literature by Dessel, et al. [5].

However, the use of robotic surgery has blossomed in community hospitals. This is the first case report of a robotic assisted resection of a mediastinal parathyroid in a community hospital setting. This is a sentinel paper documenting the feasibility of incorporating minimally invasive and inno-

*Corresponding author: Dr. Emiy Ann Harris, MD, Department of General Surgery, Amita Saint Joseph Hospital Chicago, IL60613, USA

Accepted: September 01, 2020

Published online: September 03, 2020

Citation: Harris EA, Abadin SS, Bowersox KD (2020) A Case Report of a Robotic Thoracosopic Parathyroidectomy in a Community Hospital. J Surgical Endocrinol 2(1):36-39 


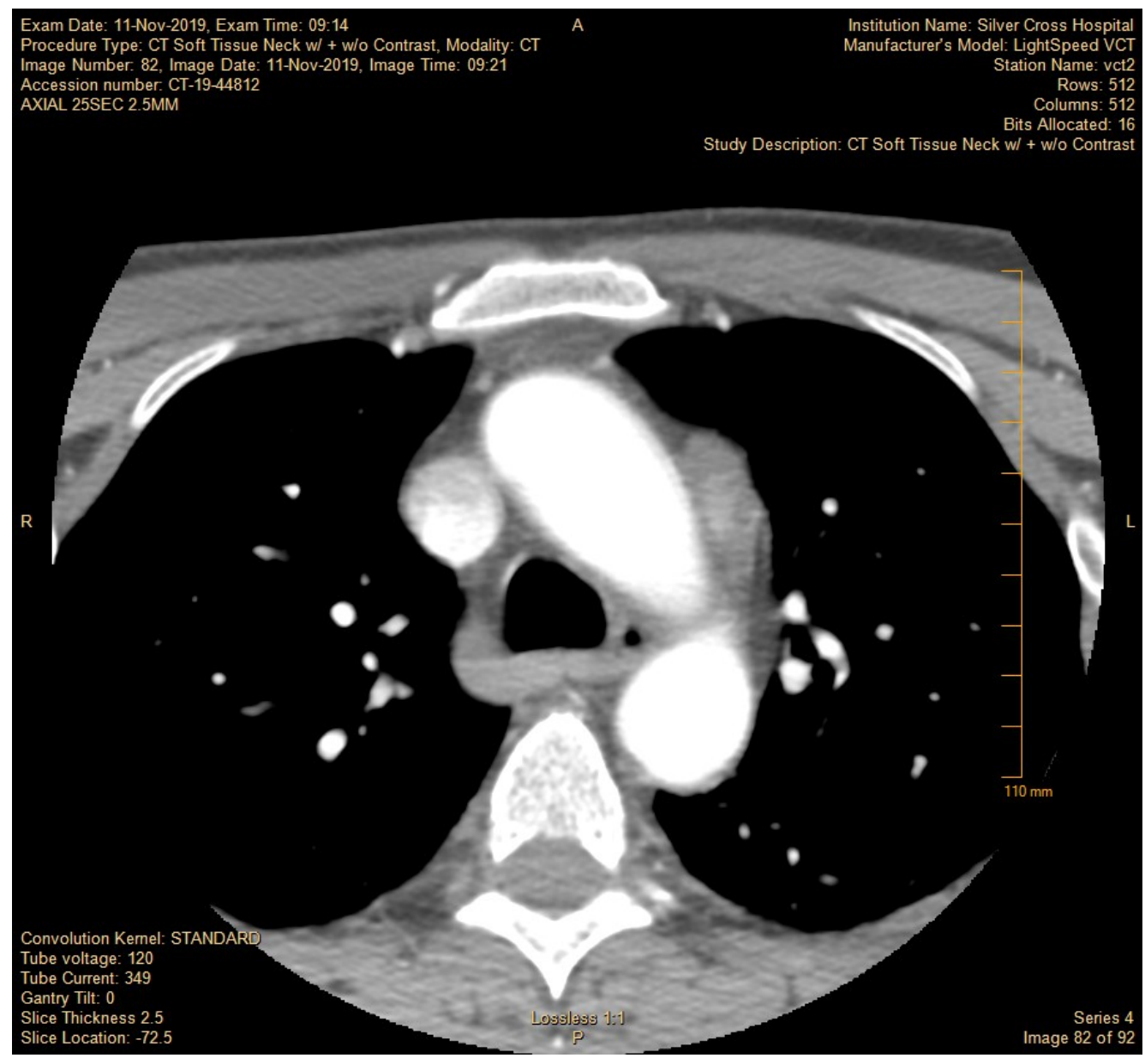

Figure 1: CT scan demonstrating aortopulmonary window pituitary adenoma.

vative surgical techniques broadly using the da Vinci system in community hospitals and not just exclusive to highly academic centers. And thereby alludes to the future of ectopic mediastinal parathyroid surgery in which robotic assistance will become the standard of care and thereby forego older traditions of sternotomy, thoracotomy, and even VATS.

\section{Case Presentation}

This is a 57-year-old man with a history of primary hyperparathyroidism status post-parathyroidectomy in 11/2019 in which an abnormal right superior parathyroid gland (adenoma on final pathology) was excised during a 4-gland exploration; however, the patient's PTH levels never normalized post-operatively. Two normal (right inferior, left superior) parathyroid glands were identified. The patient had a subsequent CT scan of the chest (Figure 1) demonstrating an aortopulmonary window mass; a subsequent SPECT/CT (Figure 2) with venous sampling was performed which demonstrated avidity and localization of the mass seen on CT imaging (Figure 1) along the aortic arch suggestive of an intrathoracic parathyroid adenoma. A sestamibi scanned (Figure 3) was performed which confirmed the suspicion that the aortopulmonary mass was indeed likely a parathyroid adenoma. The decision was made to take the patient to surgery for robotic assisted mediastinal parathyroid resection. Thoracic surgery was consulted to join the case considering the intrathoracic

\section{location of the parathyroid adenoma.}

The patient underwent fiberoptic laryngoscopy which demonstrated normal vocal cord function with good bilateral adduction and abduction followed by robotic assisted thoracoscopic mediastinal mass resection (left inferior parathyroidectomy), partial thymectomy, and intraoperative parathyroid hormone monitoring with intraoperative PTH levels dropping $83 \%$ from pre-excision. Specimens were taken intraoperative: partial thymus, mediastinal mass adjacent to aortic arch (frozen section biopsy confirmed the mass to be adenomatous parathyroid tissue).

Final pathology revealed left inferior parathyroid gland to be consistent with an adenoma or hyperplasia with hypercellularity with fibrosis and hemorrhage $(4.7 \times 2.2 \times 1 \mathrm{~cm})$. Thymus pathology revealed benign thymic tissue.

In regard to the specifics of the robotic approach for this case; the patient was placed in the right lateral decubitus position with right single lung ventilation. A $10 \mathrm{~mm}$ port endoscope was placed in the left $6^{\text {th }}$ intercostal space in anterior axillary line, and two $8 \mathrm{~mm}$ operating robotic ports placed in $4^{\text {th }}$ intercostal space a hand breadth right and left of the first incision. An accessory port with the Airseal device is placed in the midclavicular line through the sixth intercostal space through which a flexible retractor holding the lung away was placed. 


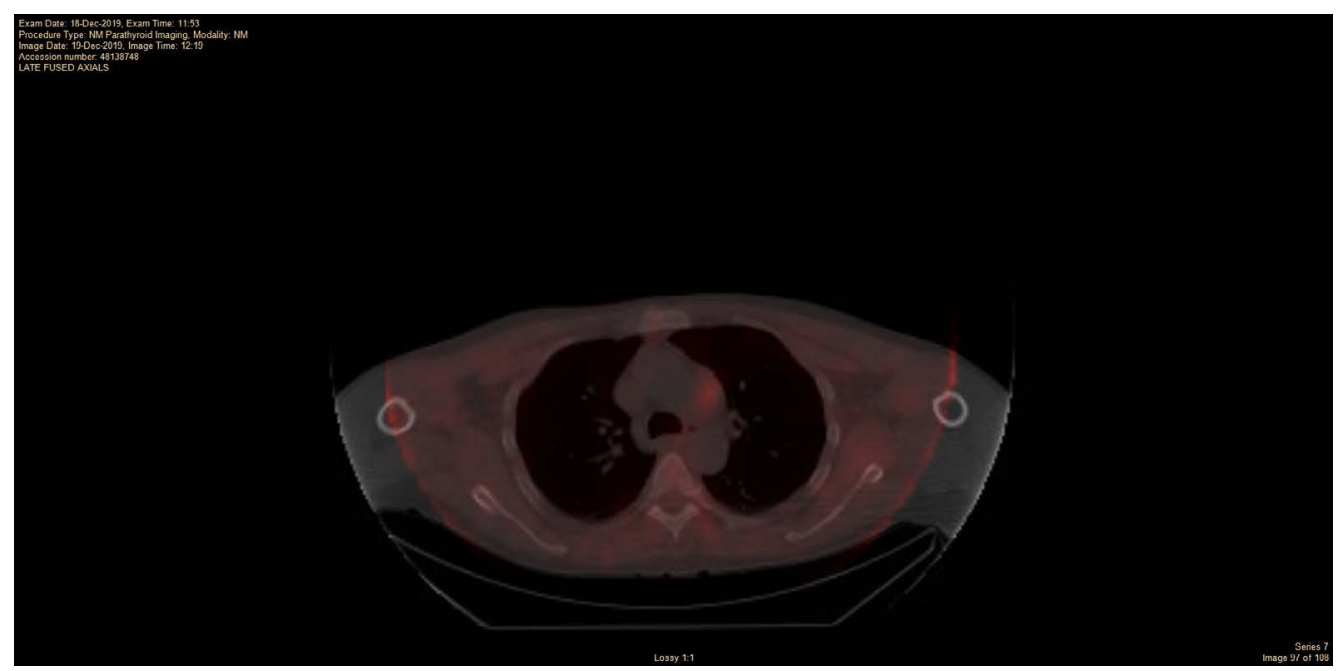

Figure 2: SPECT/CT with venous sampling demonstrating avidity of aortopulmonary window mass concerning for intrathoracic parathyroid.

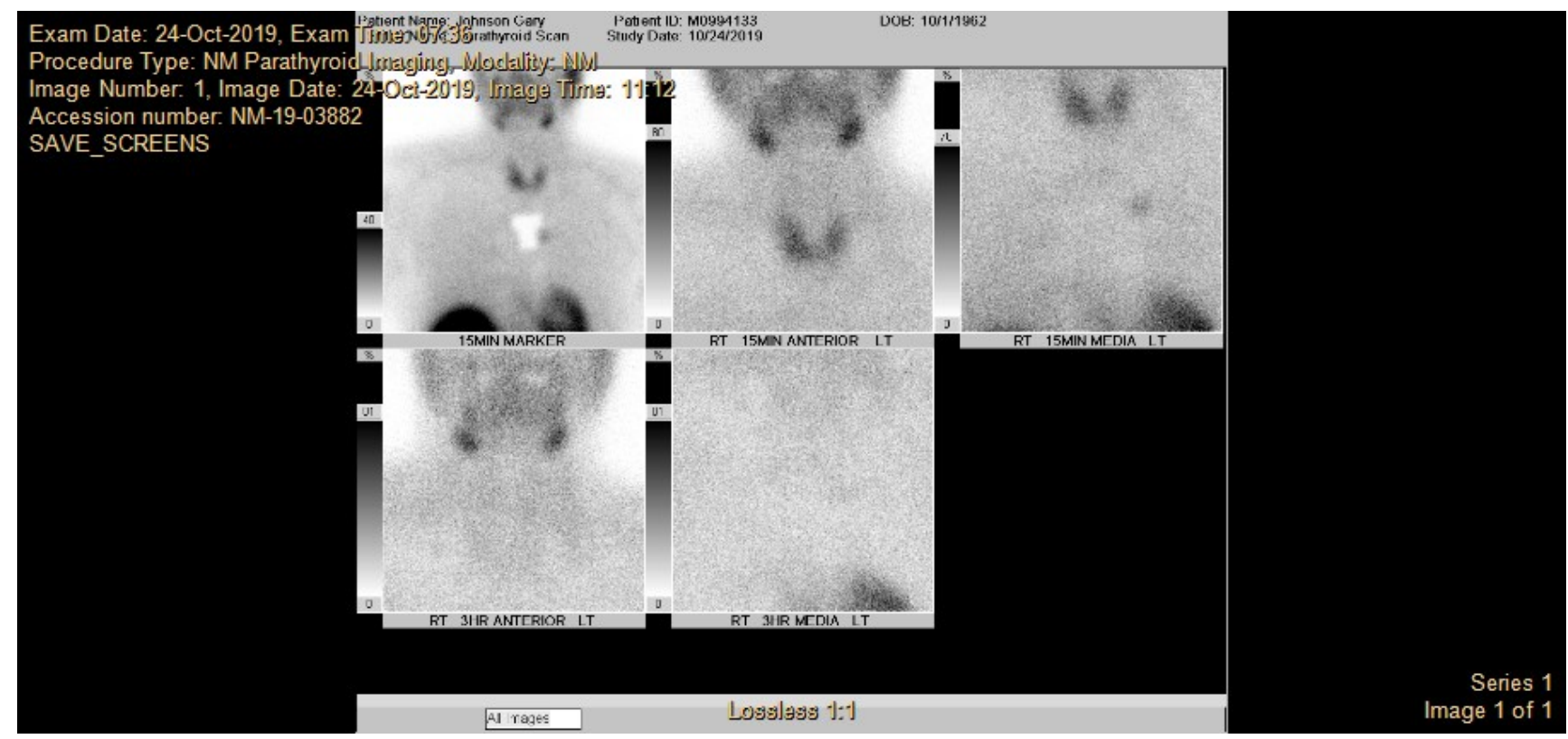

Figure 3: Sestamibi scan demonstrating activity of aortopulmonary window parathyroid adenoma.

\section{Discussion}

The resection of a parathyroid from the aortopulmonary window is an ideal case for the robotic da Vinci; with less morbidity than sternotomy and thoracotomy and with greater safety and accuracy than conventional VATs.

The use of sternotomy or thoracotomy for the treatment of mediastinal hyperparathyroidism should be regarded as a way of the past. One study from 1983 of 26 patients with primary hyperparathyroidism documented 6 sternotomies and 1 thoracotomy for this management [4]. Another study from 1962-1987 documented 36 cases of mediastinal exploration by sternotomy for treatment of hyperparathyroidism after one or more negative cervicotomies [9]; exploration was positive 20 times and negative 16 times [9]; of note in this case report 13 cases were noted to by intra thymic and 3 cases were noted to be in the middle mediastinum, which alludes to the failure to detect deeper mediastinal lesions via standard open approaches. Therefore, in consideration of historic pitfalls as well as the morbidity of sternotomy and thoracotomy, the robotic approach for mediastinal parathyroid adenomas should be the new standard of care especially when the culprit ectopic adenoma lies in the middle or deeper mediastinum.

Prior literature has proven the success for the robotic approach for resection of mediastinal lesions. One paper spanning 2001-2005 studied 33 patients with various mediastinal lesions having $91 \%$ success of using the da Vinci robot for resection [8], with the most common complication being $33 \%$ with a transient palsy of the left recurrent laryngeal nerve [8]. To date there has been no high-powered study of the robotic resection ectopic mediastinal parathyroid adenomas. 
The robotic da Vinci is advantageous in surgical resection of ectopic parathyroids and other mediastinal lesions as it affords access to tiny, remote, narrow, and difficult to reach anatomical locations, that are characteristic of the mediastinum $[7,8]$.

Furthermore, the time devoted to these robotic assisted mediastinal ectopic parathyroid excisions is reasonable and ranges from 58 [7] minutes to 130 minutes. The first robotic assisted resection of an ectopic parathyroid adenoma in the AP window recorded by Bodner was noted to last 130 mintues [3].

Lastly, the expedited recovery of patients undergoing mediastinal surgery for ectopic parathyroids is quite minimal documented in the literature to being 3 days [7], in comparison to the post-operative stay after sternotomy or thoracotomy.

Moreover, this paper demonstrates the ability of a community hospital to implement robotic minimally invasive approaches for ectopic mediastinal adenomas in the aortopulmonary window; an approach that culturally is assumed to be only accessible at academic institutions.

To speak to this high translatability of implementing robotic surgical interventions in community hospitals, one study of 101 robotic general surgery cases in a community hospital during 2014-2015 documented 99 surgeries completed robotically with 8 complications (7.92\%), with a mean operative time of 233 minutes [10]. However, there has yet to be documentation of a robotic resection of a mediastinal parathyroid adenoma in a community hospital, as this paper documents.

Therefore, this paper highlights the translatability and potential of utilizing robotic thoracascopic approaches for excision of these mediastinal lesions and thereby suggests the potential for community hospital use of the da Vinci robot for robotic mediastinal parathyroid excisions.

\section{References}

1. Makay Ö, Durmaz SF, Özdemir M, et al. (2018) Robot-assisted endoscopic mediastinal parathyroidectomy. Turk J Surg 34: 315318.

2. Harvey A, Bohacek L, Neumann D, et al. (2011) Robotic thoracoscopic mediastinal parathyroidectomy for persistent hyperparathyroidism: Case report and review of the literature. Surg Laparosc Endosc Percutan Tech 21: e24-27.

3. Bodner J, Profanter C, Prommegger R, et al. (2004) Mediastinal parathyroidectomy with the da Vinci robot: Presentation of a new technique. J Thorac Cardiovasc Surg 127: 1831-1832.

4. Roslyn JJ, Gordon HE, Mulder DG (1983) Mediastinal parathyroid adenomas. A cause of persistent hyperparathyroidism. Am Surg 49: 523-527.

5. Van Dessel E, Hendriks JM, Lauwers P, et al. (2011) Mediastinal parathyroidectomy with the da Vinci robot. Innovations (Phila) 6: 262-264.

6. Chan AP, Wan IY, Wong RH, et al. (2010) Robot-assisted excision of ectopic mediastinal parathyroid adenoma. Asian Cardiovasc Thorac Ann 18: 65-67.

7. Ismail M, Maza S, Swierzy M, et al. (2010) Resection of ectopic mediastinal parathyroid glands with the da Vinci robotic system. Br J Surg 97: 337-343.

8. Augustin F, Schmid T, Bodner J (2006) The robotic approach for mediastinal lesions. Int J Med Robot 2: 262-270.

9. Dubost Cl, Bouteloup PY (1988) Mediastinal exploration by sternotomy in surgery of hyperparathyroidism. 36 cases. J Chir (Paris) $125: 631-637$.

10. Oviedo RJ, Robertson JC, Alrajhi S (2016) First 101 Robotic General Surgery Cases in a Community Hospital. JSLS 20. 